\title{
ATLAS status and highlights
}

\author{
Fabiola Gianotti*i \\ CERN \\ E-mail: fabiola.gianotti@cern.ch
}

The ICHEP conference held in Paris in July 2010 marks an historical moment for particle physics: preliminary physics results from the LHC experiments were presented for the first time.

The CERN Large Hadron Collider (LHC) started operation at the unprecedented centre-of-mass energy of $\sqrt{s}=7 \mathrm{TeV}$ on 30 March 2010. This paper describes first physics results obtained by ATLAS. They are based in many cases on the full data sample recorded between 30 March 2010 and the beginning of ICHEP (integrated luminosity $\sim 340 \mathrm{nb}^{-1}$ ). They cover first measurements of the jet, $W$, and $Z$ production cross sections, observation of top-quark candidates, and first searches beyond the sensitivity of the Tevatron.

35th International Conference of High Energy Physics

July 22-28, 2010

Paris, France

\footnotetext{
* Speaker.

${ }^{\dagger}$ Representing the ATLAS Collaboration.
} 


\section{Introduction}

The start-up of the LHC operation at $\sqrt{s}=7 \mathrm{TeV}$ on 30 March 2010 opened the exploration of a new energy regime and a bright era for particle physics.

This paper reports first physics results obtained by the ATLAS experiment, based on an integrated luminosity of up to $\sim 340 \mathrm{nb}^{-1}$ recorded between 30 March and 19 July 2010 .

The first presentation of physics results at a prestigious international conference like ICHEP represents the crowning achievement of 20 years of efforts of the worldwide ATLAS Collaboration to build, commission and operate a detector of unprecedented technology, complexity and performance. The Collaboration consists today of about 3000 scientists (of which about 1000 are $\mathrm{PhD}$ students) from 174 Institutions and 38 countries.

This paper is organised as follows. Section 2 describes the experiment status and operation with first data. Sections 3 and 4 discuss a few examples out of a large amount of detector performance and physics results presented at ICHEP (much more information can be found in the contributions to this conference by ATLAS speakers [1]). Finally, Section 5 is devoted to the conclusions.

\section{Experiment status and operation}

The ATLAS detector and its performance are described in detail in references [2, 3]. Briefly, the inner detector consists of silicon pixels and strips (arranged in layers in the barrel part and in disks in the end-cap regions) surrounded by a transition radiation detector made of straw-tube type drift chambers. This whole system is immersed in a 2 Tesla field provided by a thin superconducting solenoid located in front of the electromagnetic calorimeter. The latter is a fine-grained lead-liquid argon sampling detector with accordion shape. Hadron calorimetry includes an ironscintillating tiles calorimeter in the central part, and liquid-argon detectors with copper and tungsten absorbers in the end-cap and forward regions (where the radiation levels are higher). Finally, the external muon spectrometer consists of gas chambers in air, immersed in strong magnetic fields provided by three superconducting toroid magnets.

Thanks to very strict construction specifications and rigorous quality controls, as well as to an intense commissioning campaign with cosmics rays in 2008 and 2009, the fraction of non-working channels in the various detector components was typically a few permil for the data samples used for ICHEP. Also large (97\% or larger in most detector systems) was the fraction of good quality data, i.e. data usable for physics analyses. These are excellent achievements for an experiment in the early stages of operation.

Figure 11 shows the integrated luminosity as a function of time delivered by the LHC in conditions of stable beams and recorded by ATLAS. By the time of the ICHEP conference, ATLAS had collected about $340 \mathrm{nb}^{-1}$ with the full detector active, corresponding to a remarkable data-taking efficiency of about $95 \%$. It can be seen that most of this luminosity was delivered in the first half of July, after the machine commissioned multi-bunch operation at high intensity (50 bunches per beam, with $10^{11}$ protons per bunch, [4]). The data have therefore been analysed very quickly and efficiently. The peak luminosity reached $\sim 1.6 \times 10^{30} \mathrm{~cm}^{-2} \mathrm{~s}^{-1}$ in the first half of July 2010 . 

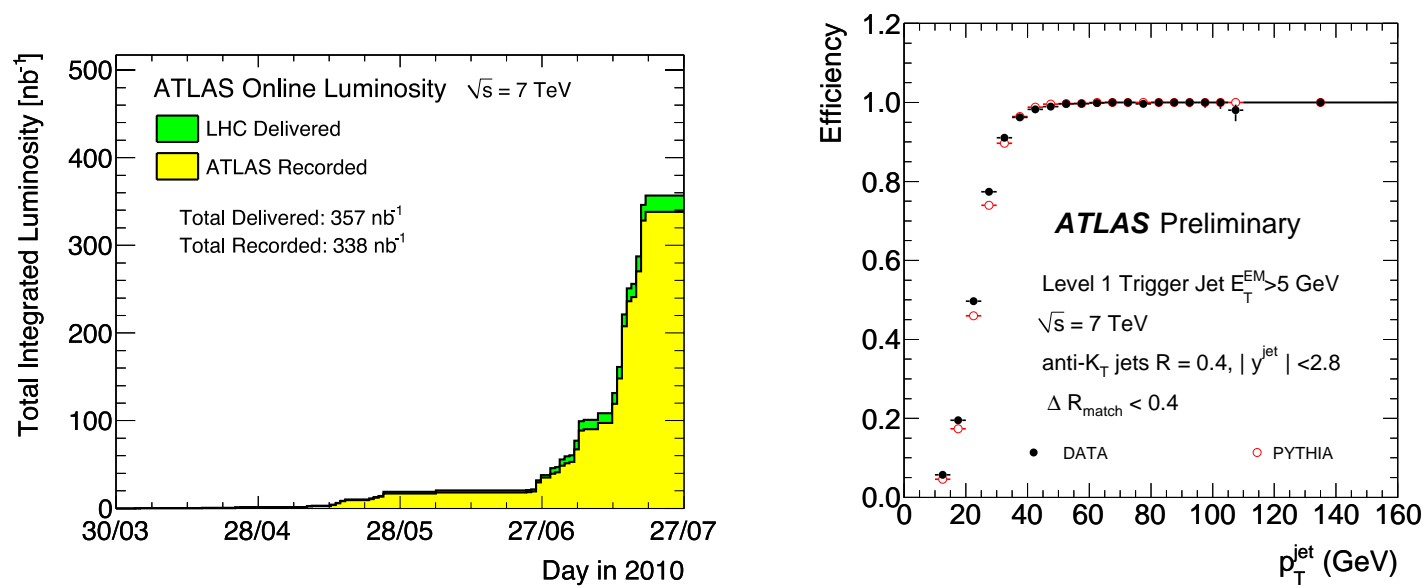

Figure 1: Left: Integrated luminosity delivered by the LHC (green) and recorded by ATLAS (yellow) from the beginning of data-taking at $\sqrt{s}=7 \mathrm{TeV}$ to the beginning of ICHEP. Right: Efficiency of the LVL1 jet trigger, as a function of the calibrated jet $p_{T}$ reconstructed offline, for the lowest threshold. The dots indicate the data and the open circles the Monte Carlo simulation.

Commissioning the trigger is one of the most crucial operations in the early stages of a hadroncollider experiment. It can also be very challenging if the machine instantaneous luminosity ramps up quickly. ATLAS has a three-level trigger system selecting about $200 \mathrm{~Hz}$ of interesting events for recording to mass storage, out of $40 \mathrm{MHz}$ (at LHC design operation) of beam-beam collisions. It is based on programmable hardware devices at level-one (LVL1), which use the fast signals from two walls of minimum-bias scintillator counters (located in front of the end-cap cryostats at $z= \pm 3.5 \mathrm{~m}$ from the collision centre), the calorimeters, and the muon spectrometer, and on software algorithms at the higher levels (HLT). As long as the instantaneous luminosity was well below $\sim 10^{29} \mathrm{~cm}^{-2} \mathrm{~s}^{-1}$ events have been selected using the LVL1 trigger only. The HLT system was run in transparent mode for commissioning purposes, without applying active rejections. When the luminosity approached $\sim 10^{29} \mathrm{~cm}^{-2} \mathrm{~s}^{-1}$, several HLT chains (reconstructing $e, \gamma$, jets, $\tau, \mu$ candidates) had to be enabled, one after the other, in order to cope with the increasing event rate while keeping low energy thresholds at LVL1. At the time of ICHEP, ATLAS had already deployed a quite rich trigger menu.

An example of (early) trigger performance is presented in figure 1 which shows the efficiency turn-on curve of the LVL1 jet trigger for the lowest available LVL1 energy threshold. The jet trigger becomes fully efficient for $p_{T} \sim 50 \mathrm{GeV}$. The data are well described by the Monte Carlo (MC) simulation, indicating a good understanding of the detector and trigger behaviour.

After being recorded in the pit, the data are processed at CERN, the "Tier-0" of the LHC Computing Grid (LCG [5]), and then distributed worldwide to the ATLAS Tier-1 and Tier-2 sites. The total throughput of ATLAS data (including MC samples and detector data) over the Grid has reached peaks of $10 \mathrm{~GB} / \mathrm{s}$, well above the design value of $2 \mathrm{~GB} / \mathrm{s}$. More than 1000 ATLAS physicists have been doing analysis using Grid resources in June-July 2010, for a total of about 11 million processed analysis jobs. The LHC Grid has been performing remarkably well right from the beginning of the LHC operation, allowing the experiments to produce physics results very 

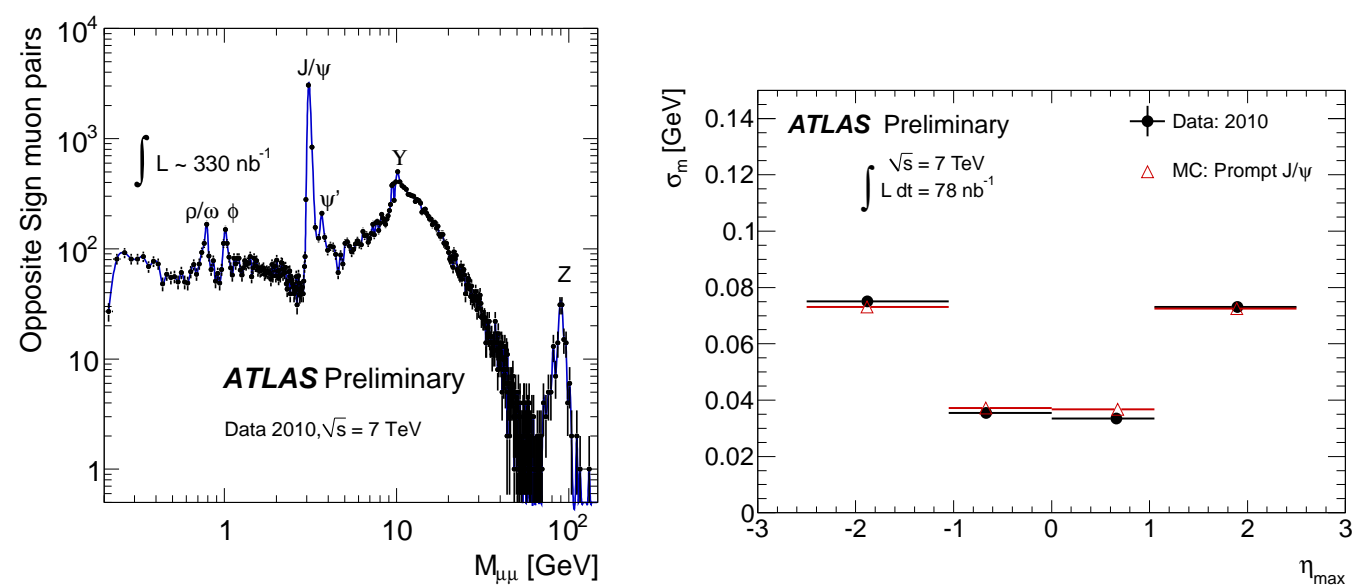

Figure 2: Left: Reconstructed mass spectrum of opposite-sign muon pairs in a data sample selected with a low-threshold LVL1 muon trigger. Right: Mass resolution of the reconstructed $J / \psi \rightarrow \mu^{+} \mu^{-}$peak, as a function of the pseudorapidity of the furthest forward muon, for the data (dots with error bars) and the simulation (open triangles).

quickly.

\section{Detector performance with first LHC data}

One of the most impressive outcomes of the first months of operation is the excellent performance of the accelerator and detectors.

As mentioned in the previous section, ATLAS has been taking data with very high efficiency and negligible fraction of non-operating channels. In addition, the detector performance is much better than expected at this early stage for a very complex apparatus, and is close to specifications in many cases.

Another very positive news is that the detector simulation [6], based on the Geant 4 toolkit [7], reproduces the detector response remarkably well, indicating a very accurate geometry description and an extremely mature understanding of the underlying physics. Clearly, 15 years of test-beam activities and increasingly realistic simulation studies to understand the detector response in all details, model it in the simulation framework and validate the simulation against real data paid dividends.

A few examples are discussed below.

The left panel in figure 2 shows the reconstructed mass spectrum for opposite-sign muon pairs in the mass range from a few hundreds $\mathrm{MeV}$ to about $100 \mathrm{GeV}$. Very simple, minimal requirements were applied to select these events: a firing LVL1 muon trigger with threshold $p_{T} \sim 6 \mathrm{GeV}$, and two opposite-sign muons, reconstructed offline by combining the information of the inner detector and the muon spectrometer, originating from within $1 \mathrm{~cm}$ from the primary vertex along the beam axis (to reject the background from cosmic rays). Several resonances are visible in the reconstructed spectrum: unresolved $\rho$ and $\omega$ peaks at $m\left(\mu^{+} \mu^{-}\right) \simeq 780 \mathrm{MeV}, \phi(1020)$, charmonium states $(J / \psi$ and $\left.\psi^{\prime}\right), Y$ and $Z$. 

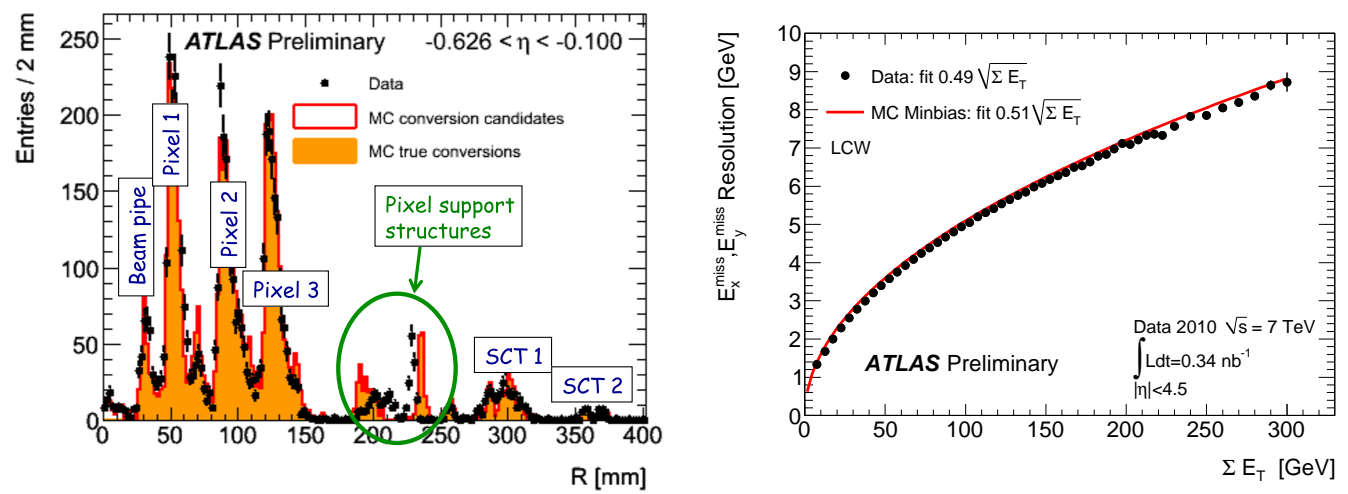

Figure 3: Left: Reconstructed conversion radius of $\gamma \rightarrow e^{+} e^{-}$candidates in the inner detector for the data (dots) and the MC simulation (open histogram). The full histogram shows the distribution for true photon conversions predicted by the simulation. The labels indicate the location of the beam pipe, active detector layers (pixels and SCT) and support structures. Data and simulation are normalized to the same number of photon conversions. Right: Resolution of the two components $\left(E_{x}^{\text {miss }}\right.$ and $\left.E_{y}^{\text {miss }}\right)$ of the missing momentum vector in the transverse plane as a function of the total transverse energy in the calorimeters, as measured in a sample of minimum-bias events. Energies are calibrated to the correct scale. The dots indicate the data points and the curve the result of a fit to the simulation. The fit results for both data and simulation are indicated.

Among the low-mass resonances, $J / \psi \rightarrow \mu^{+} \mu^{-}$plays a special role for the detector commissioning with early data, as it provides large and clean samples of low- $p_{T}$ muons to study the muon trigger, reconstruction and identification efficiency, as well as the inner detector momentum resolution and absolute scale in the few $\mathrm{GeV}$ region. The right panel in figure 2 shows the mass resolution of the reconstructed $J / \psi$ peak as a function of the muon pseudorapidity. The resolution is typically $\sim 30 \mathrm{MeV}$ in the barrel region, deteriorating to $\sim 70 \mathrm{MeV}$ in the end-cap region (mainly because of the larger amount of material). The simulation reproduces the data to a few percent. Similar studies show that the reconstructed $J / \psi$ peak is within $3 \mathrm{MeV}$ of the PDG [8] value over the full coverage of the inner detector $(|\eta|<2.5)$, and is reproduced by the simulation to better than $\sim 5 \mathrm{MeV}$. These results indicate that the absolute momentum scale of the inner detector is already understood to the permil level in the few $\mathrm{GeV}$ region.

One of the key contributions to this result is the good knowledge (already at this stage) of the material in the inner detector, as low-momentum particles are significantly affected by multiple scattering. In general, mapping the tracker material with high accuracy (few percent) is one of the most important requirements for the LHC physics programme, e.g. for a precise measurement of the $W$ mass. Several methods with complementary sensitivity will be used to achieve this goal. One of them is based on the reconstruction of converted photons. The left panel in figure 3 shows the reconstructed conversion radius for $\gamma \rightarrow e^{+} e^{-}$candidates in the ATLAS inner detector. Clear, distinct peaks are visible at the locations of material layers: the beam-pipe, three pixel layers, some pixel support structure, the first two silicon-strip (SCT) layers, etc. The data are well reproduced by the MC simulation, except in the region of the pixel support structure, where material displacements at the level of $\sim 1 \mathrm{~cm}$ are observed in the simulation. This problem, which has been fixed since then, demonstrates that the quality of the data and of the reconstruction algorithms were already 
sensitive enough to spot tiny imperfections in the simulated geometry (at the level of a percent of a radiation length in this case). Using photon conversions and other methods (e.g. the reconstruction of secondary vertices of hadronic interactions), the knowledge of the inner detector material had achieved a precision of about $10 \%$ at the time of the ICHEP conference.

Early data have also been used to understand the quality of the missing transverse energy, which is measured from the energy deposited in the calorimeters. At this stage of the experiment, this observable is a very powerful debugging tool, as it is sensitive to instrumental effects in the calorimeters (noise, coherent noise, mis-calibrations, cracks in the acceptance, etc.) and to cosmic rays and beam-related backgrounds. In the discovery era, missing transverse energy will become an excellent signature for new physics (e.g. Supersymmetry), provided the above-mentioned effects have been well understood and corrected for. The resolution of the two components of the missing momentum vector in the transverse plane is shown in the right panel of figure 3 as a function of the total transverse energy deposited in the calorimeters. The data follow the expected trend for the resolution curve and are in excellent agreement with the simulation. This is a very encouraging result, as this measurement is made over almost the full calorimetry coverage (nine pseudorapidity units), five different detectors, and about 200000 readout channels. It demonstrates that the main instrumental effects are under control.

\section{Preliminary physics results}

This section presents some highlights of the physics achievements of ATLAS in the first few months of LHC operation. They demonstrate that, already at this early stage, the "rediscovery" of the Standard Model is well advanced, and that the experiment is on good track to undertake sensitive searches for new physics. More details can be found in [1].

\subsection{Jet measurements}

Jets are the most abundant high- $p_{T}$ objects produced at hadron colliders, and have allowed the LHC experiments to exceed the Tevatron kinematic reach very quickly. This is demonstrated by the plot in the left panel of figure 4 , which shows that the invariant mass of the two leading jets in the event (i.e. the centre-of-mass energy of the parton-parton hard-scattering) extends beyond $2 \mathrm{TeV}$ (the centre-of-mass energy of the proton-antiproton collisions at the Tevatron).

The first ATLAS measurement of the inclusive jet cross section is shown in the right panel of figure 4 . It covers a broad rapidity region $(|y|<2.8)$ and jets with $p_{T}>60 \mathrm{GeV}$. The data are compared to the prediction of Next-to-Leading-Order (NLO) perturbative QCD [10]. Good agreement is observed over five orders of magnitude. The experimental uncertainties (30-40\%) are dominated by the knowledge of the absolute jet energy scale, obtained as described below.

Jet energies have been corrected for calorimeter non-compensation, impact of upstream material and other effects by using $\eta$ - and $p_{T}$-dependent calibration factors obtained from a full Geant4based simulation of the ATLAS detector (the available data sample at the time of ICHEP was not large enough for a fully data-driven method). This procedure is justified by the large number of detailed and extensive studies made to validate the simulation against test-beam and LHC data (see section 3). As jets are complex objects, efforts have been made to achieve a precise modeling of the basic constituents (calorimeter clusters, single hadrons, tracks). Longitudinal and lateral shower 

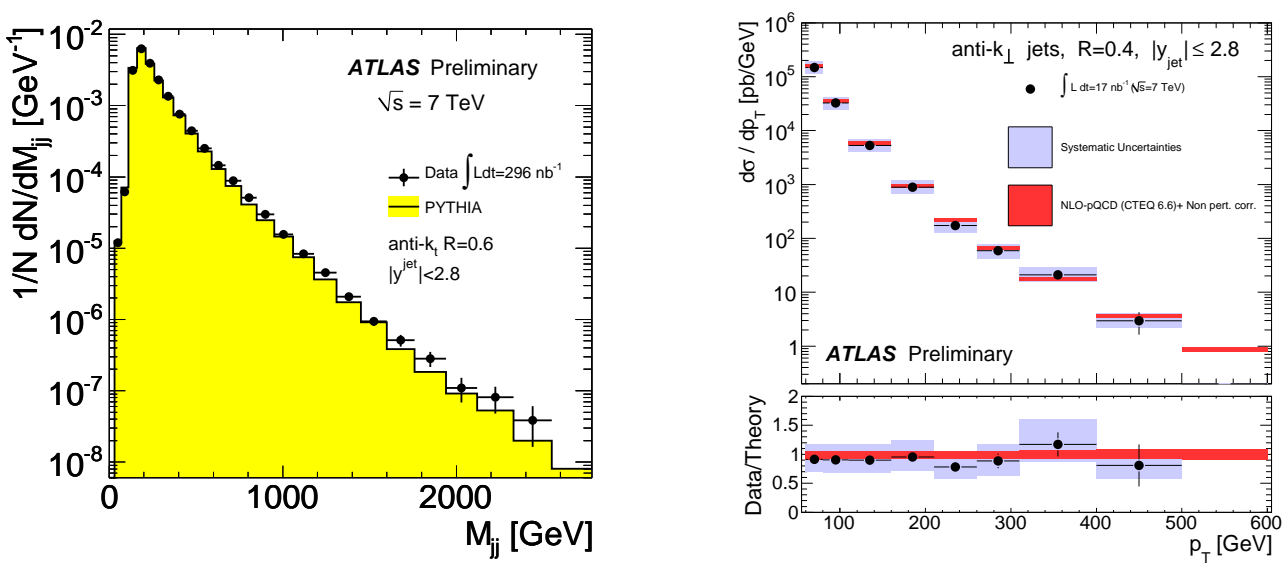

Figure 4: Left: Dijet invariant mass distributions for data (dots) and the PYTHIA [9] MC prediction (histogram), normalized to unity. The leading (sub-leading) jet is required to have $p_{T}>80$ (40) GeV. Right: Inclusive jet differential cross section (dots) as a function of the jet $p_{T}$. The error bars indicate the statistical error, and the grey bands the systematic uncertainties. The NLO perturbative QCD theoretical prediction [10] and its uncertainty are shown in red.

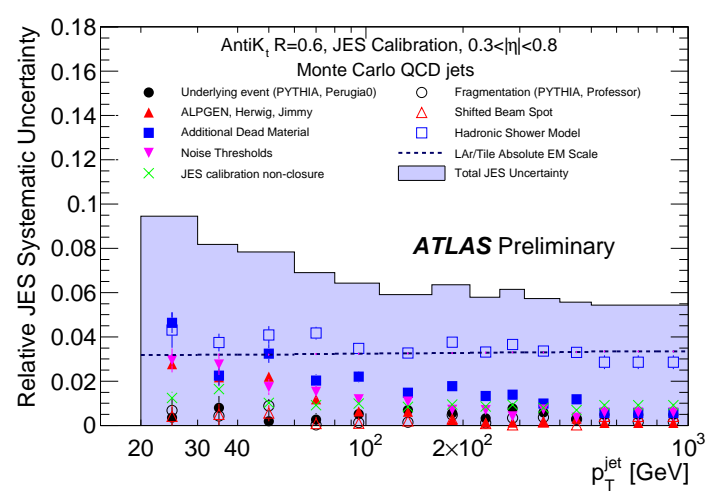

Figure 5: Fractional jet energy scale systematic uncertainty (total and the individual contributions) as a function of $p_{T}$ for jets in the central part of the barrel calorimeter.

shapes, as well as energy response variations as a function of rapidity, have also been studied in great detail. Figure 5 shows that the resulting uncertainty on the jet energy scale was at the level of $\sim 7 \%$ at the time of ICHEP. A large number of potential contributions have been studied and included: upstream material, electronic noise, shower model, MC generators, etc.

More recent results on jet physics can be found in references [11, 12, 13].

\section{2 $W$ and $Z$ measurements}

First measurements of $W$ and $Z$ bosons are fundamental milestones in the "rediscovery" of the Standard Model at $\sqrt{s}=7 \mathrm{TeV}$. These particles are also omni-present backgrounds to searches for new physics.

To select $W \rightarrow \ell v$ candidates, events were required to have a well-identified lepton (electron or muon) with $p_{T}>20 \mathrm{GeV}$, missing transverse energy $E_{T}^{\text {miss }}>25 \mathrm{GeV}$ (coming from the escaping 

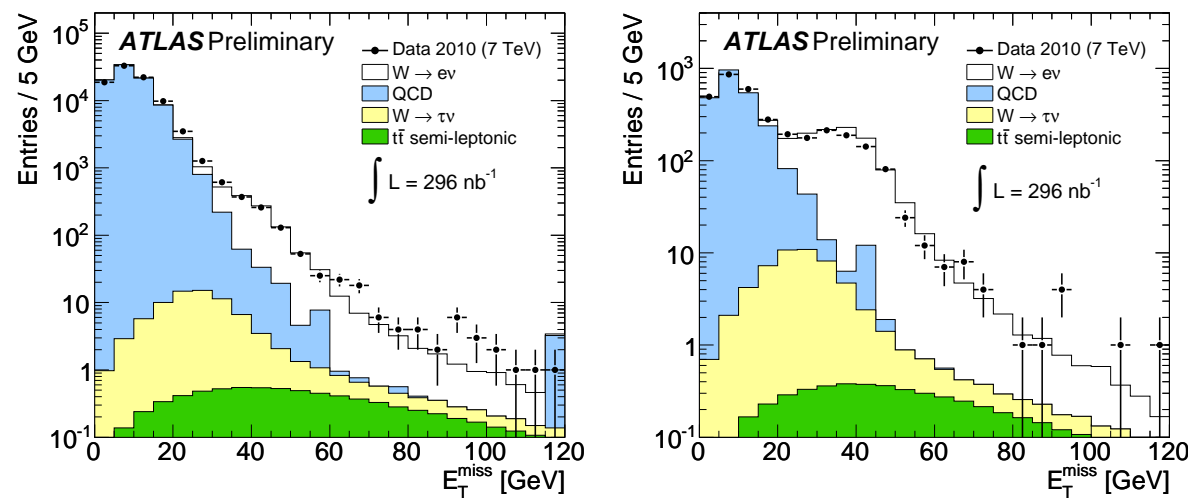

Figure 6: Missing transverse energy distributions for $W \rightarrow e v$ candidates at the pre-selection level (left panel) and after all cuts except the $E_{T}^{\text {miss }}$ and $m_{T}$ cuts (right panel). The data (dots) are compared to the PYTHIA MC prediction (histograms) for the $W \rightarrow e v$ signal and the various background components. The total number of MC events is normalised to the number of candidates observed in the data.

neutrino) and a transverse mass of the lepton-"neutrino" system $m_{T}>40 \mathrm{GeV}$. The efficiency of these selections is $30-40 \%$. A total of $815 \mathrm{~W} \rightarrow e v$ and $1111 \mathrm{~W} \rightarrow \mu v$ candidates were selected in the full data sample recorded before ICHEP. Backgrounds amount to 5-10\% of the signal. QCD jet production, which is the dominant background contribution to the $W \rightarrow e v$ process, and is not well modeled by the MC simulation, was estimated with partially data-driven methods using control samples selected in background-enhanced regions.

Figure 6 shows the $E_{T}^{\text {miss }}$ distributions for the $W \rightarrow e v$ channel at the pre-selection level (left panel), where looser lepton identification cuts are applied, and after all selections except the $E_{T}^{\text {miss }}$ and $m_{T}$ cuts (right panel). The dramatic emergence of a "Jacobian peak" in the region around $\sim 40 \mathrm{GeV}$ is observed when applying stronger identification cuts to extract genuine electrons from the background of fakes. There is good agreement in shape between the data and the PYTHIA MC prediction.

The first measurement of the $W \rightarrow \ell v$ cross section in ATLAS is compared in figure 7 with previous measurements and with the Standard Model prediction. The measured value, $\sigma(W \rightarrow \ell v)=$ $9.3 \pm 0.9$ (stat) \pm 0.6 (syst) \pm 1.0 (lumi) nb, averaged over both channels $(e, \mu)$, is a factor about four larger than at the Tevatron and is in good agreement with the Standard Model expectation. The total uncertainty of about $15 \%$ receives similar contributions from the statistical uncertainty, the systematic uncertainty (dominated by the lepton trigger, reconstruction and identification efficiencies), and the uncertainty on the integrated luminosity $(\sim 11 \%)$. The ATLAS measurements confirm an asymmetry between $W^{+}$and $W^{-}$production, with $W^{+}$featuring a $40 \%$ larger cross section, as expected in $p p$ collisions from the proton composition in terms of valence quark.

At hadron colliders the production cross section of the $Z$-boson is typically a factor $\sim 10$ smaller than the $W$ cross section. The right panel in figure 7 shows the reconstructed $Z \rightarrow e^{+} e^{-}$ mass spectrum in ATLAS. Events were selected by requiring two opposite-sign same-flavour leptons $\left(e^{+} e^{-}, \mu^{+} \mu^{-}\right)$in the final state, with $p_{T}>20 \mathrm{GeV}$. A total of 46 (79) candidates were retained in the electron (muon) channels. These samples are very pure: the signal-to-background ratio is larger than $\sim 100$ in a mass window of size $\pm 25 \mathrm{GeV}$ around the nominal $Z$ mass. At the time of 

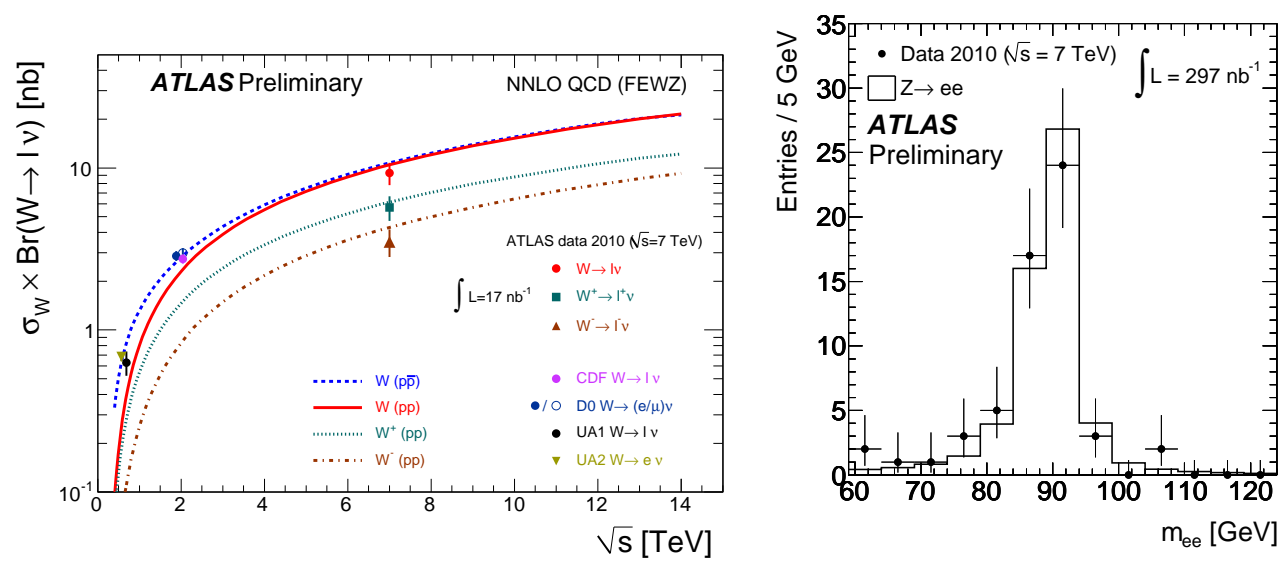

Figure 7: Left: The measured cross section for $W \rightarrow \ell v$ production (with $\ell=e, \mu$ ), and for $W^{+}$and $W^{-}$separately, compared to the theoretical predictions based on NNLO QCD calculations (FEWZ program [14]). The data points are plotted with their total uncertainty. The predictions (lines) are shown for both $p p$ collisions $\left(W^{+}, W^{-}\right.$and their sum) and $p \bar{p}$ collisions $(W)$ as a function of $\sqrt{s}$. Measurements at previous $p \bar{p}$ colliders are also shown. Right: The reconstructed $Z \rightarrow e^{+} e^{-}$mass spectrum in the data (dots) compared to the PYTHIA MC simulation (histogram). The MC is normalized to the number of events observed in the data.

ICHEP, the measured mass resolution was worse than predicted by the MC simulation (figure 7), as the inter-calibration of the electromagnetic calorimeter was at the level of 2-3\%.

The measured cross section for $66<m(\ell \ell)<116 \mathrm{GeV}$, averaged over the $e$ and $\mu$ channels, was $\sigma\left(Z / \gamma^{*} \rightarrow \ell \ell\right)=0.83 \pm 0.07$ (stat) \pm 0.06 (syst) \pm 0.09 (lumi) nb at the time of ICHEP, in good agreement with the Standard Model prediction.

More recent results on $W$ and $Z$ physics can be found in references [15, 16].

\subsection{Observation of top-quark candidates}

The $t \bar{t}$ production cross section is expected to be $\sim 160 \mathrm{pb}$ in $p p$ collisions at $\sqrt{s}=7 \mathrm{TeV}$, a factor about 20 larger than at the Tevatron. At hadron colliders the two main channels for precise measurements are the lepton+jet channel $(\ell j)$, arising from decays of the type $t \bar{t} \rightarrow b W b W \rightarrow$ $b \ell v b j j$, and the dilepton channel $(\ell \ell)$, arising from $t \bar{t} \rightarrow b W b W \rightarrow b \ell v b \ell v$. In both cases $\ell=$ $e$ or $\mu$. The $\ell j$ channel has a factor $\sim 7$ larger cross section, whereas the $\ell \ell$ channel has a better signal-to-background ratio.

A preliminary ATLAS analysis to select $\ell j$ candidates required one identified electron or muon with $p_{T}>20 \mathrm{GeV}$, at least four jets with $p_{T}>20 \mathrm{GeV}$, one of which should be tagged as coming from a b-quark, and $E_{T}^{\text {miss }}>20 \mathrm{GeV}$. To select $\ell \ell$ candidates, two opposite-sign leptons $\left(e^{+} e^{-}, \mu^{+} \mu^{-}, e^{ \pm} \mu^{\mp}\right)$ with $p_{T}>20 \mathrm{GeV}$ were required, at least two jets with $p_{T}>20 \mathrm{GeV}$ and large $E_{T}^{\text {miss }}$. In addition, in the case of $e^{+} e^{-}$and $\mu^{+} \mu^{-}$final states, the invariant mass of the lepton pair was required to be away from the $Z$ mass region. The signal efficiency of these selections is about $25-30 \%$ for both the above channels, and the expected signal-to-background ratios are $\sim 3$ in the $\ell j$ channel and $\sim 10$ in the $\ell \ell$ channel.

An analysis of this type was conducted on the full data sample recorded before ICHEP. Nine candidates were found in the data, seven in the $\ell j$ channel (four $e j$ and three $\mu j$ ) and two in the $\ell \ell$ 


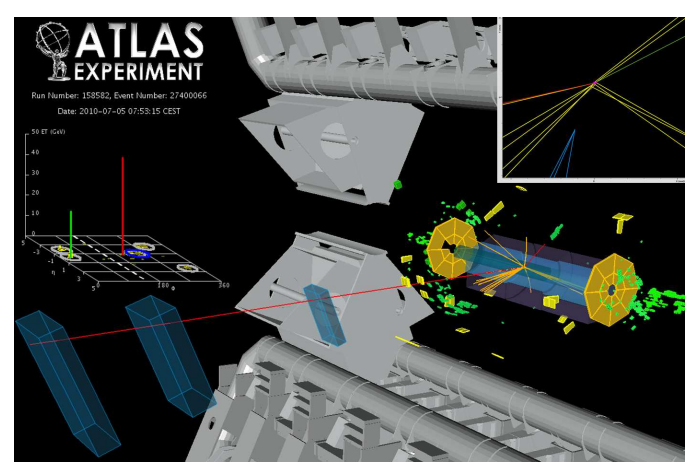

Figure 8: Display of the $t \bar{t} \rightarrow e \mu+X$ event candidate recorded by ATLAS in July 2010. The red line indicates the muon direction, while the electron is shown as a green track associated with a calorimeter cluster. The insert gives an expanded view of the vertex region, showing three tracks with $p_{T}>1 \mathrm{GeV}$ originating from the secondary vertex (in blue).

channel (one $e e$ and one $e \mu$ ). The expectation from $t \bar{t}$ production is 5 events and 0.5 events in the two channels, respectively. The kinematic properties of the 9 observed candidates are consistent with $t \bar{t}$ production.

Figure 8 shows one of the selected candidates. It is a beautiful dilepton event, containing a muon with $p_{T}=48 \mathrm{GeV}$, an electron with $p_{T}=23 \mathrm{GeV}$, and large missing transverse energy $\left(E_{T}^{\text {miss }}>77 \mathrm{GeV}\right)$. The leading jet $\left(p_{T}=57 \mathrm{GeV}\right)$ is tagged as a b-quark jet, and originates from a secondary vertex at a distance of $3.8 \mathrm{~mm}$ from the primary vertex in the transverse plane. This event is very pure, as the signal-to-background ratio for $e \mu$ events with a b-tagged jet is expected to be $\sim 15$.

This first observation of candidate events due to $t \bar{t}$ production marks the beginning of an extremely interesting era of precision physics in the top-quark sector at the LHC [3].

More recent ATLAS results on top physics can be found in reference [17].

\subsection{Searches for new physics}

Another important result of the first months of operation is that the big energy leap provided by the LHC has allowed ATLAS to perform first searches for new physics with, in some cases, sensitivity comparable with the Tevatron collider, in spite of the very small amount of luminosity accumulated before the ICHEP conference ( $\sim 340 \mathrm{nb}^{-1}$ compared to several $\mathrm{fb}^{-1}$ at the Tevatron). In one case, described below, the present Tevatron limits have been exceeded.

Strongly-produced new particles are the best candidates to break new ground, as QCD cross sections grow fast with centre-of-mass energy. Therefore, the measured dijet mass spectrum has been examined, looking for the possible production of new (heavy) resonances decaying into two jets, such as the excited quarks $q^{*} \rightarrow g q$ predicted by Compositeness theories. The left panel in figure 9 shows that the dijet spectrum measured in the data is smooth with no bumps. Upper limits were therefore set on the $q^{*}$ production cross section, which translate into lower bounds on mass. This is shown in the right panel of figure 9. The mass range $0.4<m\left(q^{*}\right)<1.29 \mathrm{TeV}$ was excluded at the $95 \%$ C.L. ${ }^{1}$ This limit includes systematic uncertainties (from e.g. the knowledge

\footnotetext{
${ }^{1}$ Masses below $0.4 \mathrm{TeV}$ are not excluded because they would contribute to a region where the dijet mass spectrum
} 

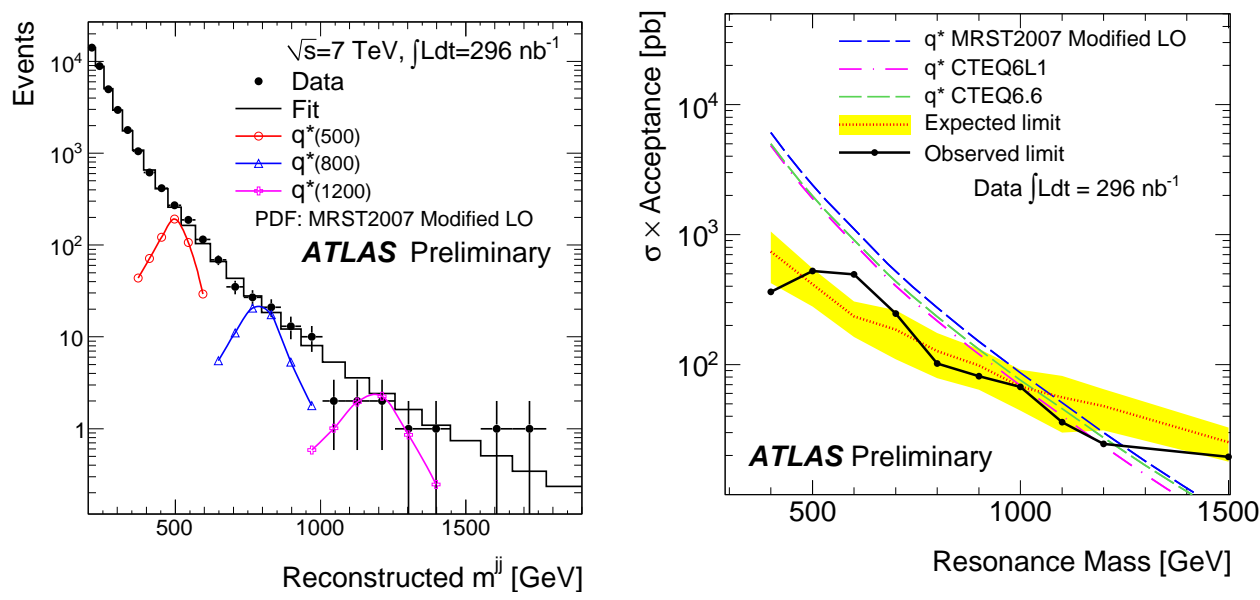

Figure 9: Left: The measured dijet mass distribution (points with error bars) for events with a leading jet with $p_{T}>80 \mathrm{GeV}$ and a sub-leading jet with $p_{T}>30 \mathrm{GeV}$. Overlaid is a fit with a smooth function (black line). The expected signals from excited quarks of various masses are also shown. Right: The measured 95\% C.L. upper limit on the accepted cross section after all selections as a function of the dijet mass (black solid curve). The red line with the yellow band shows the expected limit with its uncertainty. The dashed curves give the expected accepted cross sections for $q^{*}$ production for different choices of the Parton Distribution Functions (PDF). The $q^{*}$ mass lower bound is set where the measured limit (black curve) intersects the predicted cross section.

of the jet energy scale and luminosity, the fit to the dijet mass spectrum) and deteriorates by only $\sim 100 \mathrm{GeV}$ when varying the PDF set from the ATLAS default (MRST2007) to a different set (CTEQ6L1). The upper bound of the excluded region $(1.29 \mathrm{TeV})$ exceeds the CDF limit $(260<$ $\left.m\left(q^{*}\right)<870 \mathrm{GeV}[18]\right)$, and is the most stringent to date.

More recent ATLAS searches for new physics in dijet final states are described in references [19, 20].

\section{Conclusions}

Since 30 March 2010 ATLAS has been recording data during the first LHC run at $\sqrt{s}=7 \mathrm{TeV}$. A total integrated luminosity of $\sim 340 \mathrm{nb}^{-1}$ has been collected until the beginning of the conference. The Collaboration is extremely grateful to the LHC operation team for an impressive machine turn-on.

The whole experiment has worked very efficiently, from data-taking at the pit (with $\sim 95 \%$ efficiency), through data calibration, processing and transfer worldwide, to the quick delivery of physics results (many of which are based on the full data sample recorded until the beginning of ICHEP).

The first data demonstrate that the detector performance, and the quality of the reconstruction and simulation software, are better than expected at this (initial) stage of the experiment, and are close to nominal in some cases.

is not fully unbiased. 
Preliminary physics results include first measurements of the jet, $J / \psi, W, Z$ production cross sections, observation of top-quark candidates, and first searches for new physics with sensitivity exceeding that of the Tevatron.

The ICHEP 2010 conference marks the beginning of the LHC physics exploitation and therefore of a new, hopefully glorious era for particle physics.

\section{References}

[1] Contributions of ATLAS speakers to these Proceedings.

[2] The ATLAS Collaboration, The ATLAS Experiment at the CERN Large Hadron Collider, JINST 3 (S08003) 2008.

[3] The ATLAS Collaboration, Expected performance of the ATLAS experiment: detector, trigger and physics, CERN-OPEN-2008-020.

[4] S. Myers, contribution to these Proceedings.

[5] I. Bird, B. Jones and K.F. Kee, The organization and management of Grid infrastructures, IEEE Computer, 42 (36) January 2009.

[6] ATLAS Collaboration, The ATLAS simulation framework, Eur. Phys. J. C 70 (823) 2010.

[7] S. Agostinelli et al., Geant4: a simulation toolkit, Nucl. Instr. and Meth. A 506 (250) 2003.

[8] K. Nakamura et al. (Particle Data Group), The Review of Particle Physics, J. Phys. G 37 (075021) 2010.

[9] T. Sjöstrand, S. Mrenna and P. Skands, PYTHIA6.4 physics and manual J. High Energy Phys. 05 (026) 2006.

[10] Z. Nagy, Next-to-leading order calculation of three jet observables in hadron hadron collision, Phys. Rev. D 68 (094002) 2003.

[11] The ATLAS Collaboration, Measurement of inclusive jet and dijet cross sections in proton-proton collisions at 7 TeV centre-of-mass energy with the ATLAS detector, Eur. Phys. J. C 71 (1512) 2011.

[12] The ATLAS Collaboration, Study of jet shapes in inclusive jet production in pp collisions at $\sqrt{s}=7 \mathrm{TeV}$ using the ATLAS detector, accepted for publication in Phys. Rev. D, [arXiv:1101.0070v1 [hep-ex]].

[13] The ATLAS Collaboration, Measurement of dijet azimuthal decorrelations in pp collisions at $\sqrt{s}=7 \mathrm{TeV}$, submitted for publication in Phys. Rev. Lett., [arXiv:1102.2696v2 [hep-ex]].

[14] C. Anastasiou, L. Dixon, K. Melnikov and F. Petriello, High-precision QCD at hadron colliders: electroweak gauge boson rapidity distributions at NNLO, Phys. Rev. D 69 (094008) 2004.

[15] The ATLAS Collaboration, Measurement of the $W \rightarrow \ell v$ and $Z \gamma^{*} \rightarrow \ell \ell$ production cross sections in proton-proton collisions at $\sqrt{s}=7 \mathrm{TeV}$ with the ATLAS detector, J. High Energy Phys. 12 (060) 2010.

[16] The ATLAS Collaboration, Measurement of the production cross section for $W$-bosons in association with jets in pp collisions at $\sqrt{s}=7 \mathrm{TeV}$ with the ATLAS detector, submitted for publication in Phys. Lett. $B$, [arXiv:1012.5382 [hep-ex]].

[17] The ATLAS Collaboration, Measurement of the top quark-pair production cross section with ATLAS in pp collisions at $\sqrt{s}=7 \mathrm{TeV}$, accepted for publication in Eur. Phys. J. C, [arXiv:1012.1792v2 [hep-ex]]. 
[18] The CDF Collaboration, Search for new particles decaying into dijets in proton-antiproton collisions at $\sqrt{s}=1.96$ TeV, Phys. Rev. D 79 (112002) 2009.

[19] The ATLAS Collaboration, Search for new particles in two-jet final states in 7 TeV proton-proton collisions with the ATLAS detector at the LHC, Phys. Rev. Lett. 105 (161801) 2010.

[20] The ATLAS Collaboration, Search for quark contact interactions in dijet angular distributions in pp collisions at $\sqrt{s}=7$ TeV measured with the ATLAS detector, Phys. Lett. B 694 (327) 2011. 